\title{
Energy-efficient MAC protocols for WBANs: Opportunities and challenges
}

Sana Ullah, Changle Li

\begin{abstract}
Wireless body area networks (WBANs) are expected to play a significant role in smart healthcare systems. One of the most important attributes of WBANs is to increase network lifetime by introducing novel and low-power techniques on the energy-constrained sensor nodes. Medium access control (MAC) protocols play a significant role in determining the energy consumption in WBANs. Existing MAC protocols are unable to accommodate communication requirements in WBANs. There is a need to develop novel, scalable and reliable MAC protocols that must be able to address all these requirements in a reliable manner. In this special issue, we attracted high quality research and review papers on the recent advances in MAC protocols for WBANs.
\end{abstract}




\title{
Energy-efficient MAC protocols for WBANs: Opportunities and challenges
}

\author{
Sana Ullah · Changle Li
}

Wireless body area networks (WBANs) are expected to play a significant role in smart healthcare systems. One of the most important attributes of WBANs is to increase network lifetime by introducing novel and low-power techniques on the energy-constrained sensor nodes. Medium access con-trol (MAC) protocols play a significant role in determin-ing the energy consumption in WBANs. Existing MAC pro-tocols are unable to accommodate communication require-ments in WBANs. There is a need to develop novel, scalable and reliable MAC protocols that must be able to address all these requirements in a reliable manner. In this special issue, we attracted high quality research and review papers on the recent advances in MAC protocols for WBANs.

We have accepted six papers that address MAC protocol design, topology adjustment, attack, and cross-layer optimization issues in WBANs. The first three articles are related to MAC protocol design in WBANs. The article entitled "HEH-BMAC: Hybrid polling MAC protocol for WBANs operated by human energy" presents a hybrid MAC protocol which combines polling and contention access methods and offers service differentiation for nodes with different priorities. Simulation results show that the proposed protocol is adaptable to changes in different parameters such as energy harvesting rates, packet inter-arrival times and network size. The article entitled "An adaptive MAC protocol for real-time and reliable communications in medical cyber-physical systems" focuses on reliability and timeliness guarantees and presents an adaptive MAC protocol. It enables dynamic GTS allocation and provides different services for different types of data. With the OMNET++ simulator, the performance gain is demonstrated and compared with IEEE 802.15.4 MAC protocol. Relay process is introduced to the WBAN MAC protocol design in the article entitled "Energy-aware adaptive topology adjustment in wireless body area networks". To support the energy-efficient transmission, the network topology changes from one-hop to multi-hop. Simulation results show the superiority of the protocol in terms of network lifetime. Multiple attacks on WBANs may also affect the network lifetime. The article entitled "A survey: Energy exhausting attacks in MAC protocols in WBANs" provides a comprehensive survey of energy exhausting attacks on MAC protocols. Analysis and summary of the effects of attacks against different MAC protocols are presented. Cross-layer optimization is another important challenge in WBANs, which is addressed by the last two articles. The article entitled "A cross-layer energy efficiency optimization model for WBAN using IR-UWB transceivers" presents an energy efficiency optimization model for IEEE 802.15.6 based WBAN taking into account physical and MAC layers. The model enables energy efficiency optimization as a function of communication distance and payload size for different code rates. In the last article entitled "A cross-layer QoS-Aware optimization protocol for guaranteed data streaming over wireless body area networks", the authors propose an adaptive routing and bandwidth allocation protocol for data streaming in WBANs. Numerical results derived from a wide range of practical scenarios show that the proposed protocol is close to the theoretical solutions.

Acknowledgments This work was partially supported by National Funds through FCT (Portuguese Foundation for Science and Technology) and by ERDF (European Regional Development Fund) through COMPETE (Operational Programme 'Thematic Factors of Competi- 
tiveness'), within project FCOMP-01-0124-FEDER-037281 (CISTER) and by the North Portugal Regional Operational Programme (ON.2 O Novo Norte), under the National Strategic Reference Framework (NSRF), through the European Regional Development Fund (ERDF) and FCT, within project ref. NORTE-07-0124-FEDER-000063 (BESTCASE, New Frontiers). We would like to thank the Editor-in-Chief for giving us the opportunity to organize this special issue. We would also like to thank the Springer staff and all the reviewers, without them this special issue would not have been successfully executed. 